\title{
La intervención socioeducativa y psicosocial en el ámbito local: aportaciones para su definición adaptada a cada municipio
}

\author{
Jesús Otaño Maiza \\ Programa Hurbildu, Servicio de Protección a la Infancia y Adolescencia, \\ Departamento de Políticas Sociales, Diputación Foral de Gipuzkoa \\ jesusotano@euskalnet.net
}

Euskal Herriko Gizarte Zerbitzuen Legea eta haren oinarrizko gizarte-zerbitzuetako - tokian tokiko eremua, zerbitzu eta prestazioen katalogoaren zabaltzea, gizarte- eta hezkuntza-arloko jarduerak zerbitzu bakar batean biltzen dira: "Gizarteeta hezkuntza-arloan eta arlo psikosozialean eskuartzeko zerbitzua" deituriko zerbitzuan. Artikulu honen asmoa zerbitzu horren eredu tekniko baten definizioan laguntzea da, udalerri bakoitzaren errealitateari egokitua eta aurrez txertatutako programa eta zerbitzuen ibilbidea kontutan hartzen duena. Hemen planteatzen diren kontsiderazioek, hausnarketa teknikoan eta praktikoan eta aholkularitza teknikoan ondorioztatzen direnak, beharren erantzuna, pertsonan zentratukoa eta komunitatearen garapena indartuko duen arretaikuspegia sustatu nahi dute; udalerri bakoitzeko eta egungo legediaren xede eta printzipioekin koherentzia mantentzen duena, azken batean, zerbitzu bat definitzea ahalbideratzeko.

\section{GAKO-HITZAK:}

Gizarte-eskuartzea, arlo psikosozialeko eskuartzea, lehen-mailako arreta, tokian tokiko eremua, zerbitzueredua, babesa, inklusioa.
La Ley de Servicios Sociales del País Vasco y el despliegue de servicios y prestaciones de su catálogo de atención primaria -de ámbito local- agrupan las actuaciones de naturaleza socioeducativa y psicosocial en un único servicio denominado Servicio de Intervención Socioeducativa y Psicosocial. Este artículo pretende contribuir a definir un modelo técnico para este servicio que se adapte a cada realidad municipal y que tenga en cuenta las trayectorias de programas y servicios ya implementados o implementados anteriormente. Las consideraciones aquí planteadas, derivadas de la reflexión técnica y de la práctica de la atención directa y el acompañamiento, buscan favorecer un enfoque de atención que responda a las necesidades, se centre en la persona y fomente el desarrollo de la comunidad, enfoque que permita construir un modelo de servicio propio en cada municipio, ajustado a la legislación actual y coherente con sus principios y finalidades.

\section{Palabras Clave:}

Intervención socioeducativa, intervención psicosocial, atención primaria, ámbito local, modelo de servicio, protección, inclusión. 


\section{Introducción}

La Ley de Servicios Sociales 12/08, aprobada por el Parlamento Vasco en diciembre de 2008 tras su largo proceso de elaboración, con participación de las instituciones y, en gran medida, de diferentes agentes sociales, apuesta por avanzar en la adecuación de los servicios sociales como servicio público a la ciudadanía, fundamentalmente, en tres aspectos:

- La adecuación de los servicios en respuesta rigurosa a las necesidades.

- La consideración de los servicios sociales elevados a la categoría de derecho subjetivo.

- La progresiva universalización de los servicios para todos y todas las ciudadanas y en todo el territorio.

La aplicación de esta ley viene, por su parte, del despliegue de dos documentos normativos fundamentales: de una parte, los servicios y prestaciones que se sustancian en el Decreto de Cartera de Prestaciones y Servicios 185/15; de otra, el Mapa de Servicios Sociales en la Comunidad Autónoma del País Vasco (CAPV), que garantiza la distribución territorial homogénea de servicios en tres ámbitos o zonas básicas (urbanas, rurales y agrupaciones), áreas de servicios sociales que, divididas en sectores, definen el ámbito territorial, cobertura, ratios, etc.

Sabemos el recorrido que la implementación de la Ley de Servicios Sociales y todo su desarrollo de servicios ha tenido en el tiempo y, en cierto modo, sigue teniendo desde su aprobación. La ausencia de una aproximación económica de gasto (memoria económica) a la hora de su puesta en marcha, las dificultades derivadas de la crisis, en su momento, y la desigual supervisión y apuesta por parte de los diferentes gobiernos para su implantación progresiva son algunas de las razones que han dificultado su pleno desarrollo.

El presente artículo trata únicamente del servicio que en la cartera se denomina Servicio de Intervención Socioeducativa y Psicosocial que, con el epígrafe 1.3, establece el de esa naturaleza en el ámbito y la competencia municipal.

Así pues, proponemos un acercamiento en clave de oportunidad a la definición de este servicio (1.3) a partir de unas consideraciones que contribuyan a la reflexión, optimización y ajuste, y permitan afianzarlo con carácter integrado dentro de un único sistema de servicios sociales. Para ello, tomamos como punto de partida, como no podría ser de otra manera, los servicios, programas y actuaciones que ya se vienen desarrollando en los diferentes municipios de la Comunidad Autónoma del País Vasco.

\section{La intervención socioeducativa y psicosocial en el marco legal del País Vasco}

Centrándonos en la Ley 12/2008, como norma general se apunta a una comprensión más amplia del bienestar social incorporando a los servicios sociales al resto de sistemas de atención (salud, educación y otros) y estableciendo una serie de principios vinculantes de cara la definición de los servicios en

\begin{tabular}{|c|c|c|}
\hline \multicolumn{3}{|c|}{ Cuadro 1. Ficha de Servicio de Intervención Socioeducativa y Psicosocial (1.3) } \\
\hline Definición y Objetivo & \multicolumn{2}{|c|}{$\begin{array}{l}\text { Este servicio consiste en un conjunto de prestaciones relacionales de apoyo socioeducativo - de carácter } \\
\text { individual, familiar y/o grupal - que pueden desarrollarse tanto en el domicilio familiar (educación doméstica, } \\
\text { intervención familiar básica) como en el entorno comunitario (educación de calle, intermediación sociocultural) } \\
\text { Sus objetivos son que las personas usuarias: a) adquieran y desarrollen capacidades (actitudes, aptitudes, } \\
\text { conocimientos, criterios, pautas, habilidades) personales, familiares o grupales, que favorezcan su desenvolvimiento } \\
\text { autónomo, su inclusión social y la adecuada convivencia en el medio familiar y comunitario; b) sustituyan hábitos, } \\
\text { conductas, percepciones, sentimientos y actitudes, que resulten inadecuados por otros más adaptados. }\end{array}$} \\
\hline \multirow{5}{*}{ Prestaciones } & \multirow{5}{*}{ De servicios sociales } & Información. \\
\hline & & Intervención socioeducativa y psicosocial: intervención educativa y/o psicosocial. \\
\hline & & Valoración de seguimiento. \\
\hline & & Acompañamiento social. \\
\hline & & Mediación-intermediación \\
\hline \multirow[t]{2}{*}{ Población destinataria } & Situación & $\begin{array}{l}\text { A. Personas en riesgo de exclusión. } \\
\text { B. Personas en riesgo de dependencia. } \\
\text { C. Personas menores de edad en situación de riesgo leve o moderado de desprotección, } \\
\text { quedando excluidas las personas en situación de riesgo de desprotección } \\
\text { grave, menores de edad o adultas, cuya atención corresponde a los servicios de } \\
\text { Intervención socioeducativa y/o Psicosocial con Familias (2.7.3.1). }\end{array}$ \\
\hline & Edad & Sin límites de edad. \\
\hline \multirow{2}{*}{ Requisitos de acceso } & Administrativos & $\begin{array}{l}\text { Los requisitos generales de titularidad del artículo } 3 \text { de la Ley de Servicios Sociales no } \\
\text { serán exigibles cuando la intervención se oriente a atender situaciones de riesgo leve o } \\
\text { moderado de desprotección que afecten a perdonas menores de edad. }\end{array}$ \\
\hline & De Necesidad & $\begin{array}{l}\text { Precisar de apoyo educativo y/o psicosocial para: a) mejorar la convivencia familiar; } \\
\text { b) mejorar las habilidades de atención y cuidado de sus miembros; c) y/o facilitar o } \\
\text { posibilitar el desenvolvimiento autónomo. }\end{array}$ \\
\hline
\end{tabular}

Fuente: Decreto de Cartera 185/15. 
general y del Servicio de Intervención Socioeducativa y Psicosocial en particular. De entre estos principios, reconocemos algunos que en su contenido conectan con elementos de la "cultura" y la tarea de los socioeducativo que señalamos a continuación:

- La integración y la autonomía como finalidad.

- El punto de vista promotor añadido a la prevención y a la protección.

- El necesario diagnóstico específico de cada situación, cada persona, cada familia y cada grupo.

- El acompañamiento social, que conlleva trabajo relacional como estrategia y método de trabajo básico y fundamental del y de la profesional de la educación social.

- El enfoque comunitario de la intervención social, la cercanía, la proximidad, la adaptación de los recursos y servicios, la continuidad de la atención y la persona de referencia.

Todos estos aspectos establecen una nueva conexión con el desarrollo de la ley, su despliegue en la cartera de servicios y la práctica de la intervención real a través de dos consideraciones: por un lado, pueden entenderse, desde su desarrollo en gran parte de las iniciativas desde lo socioeducativo, como una proyección al resto de las actuaciones desde los servicios sociales; por otro lado, deben reconocer aspectos que, en esencia, siempre han estado en el discurso de la acción social y que requieren ser recuperados e integrados a partir de un enfoque definido y unas actuaciones concretas (Ávila et al. 2017: 8-9).

Por otra parte, la ley introduce de modo descriptivo en su exposición de motivos la intervención socioeducativa como parte esencial de la intervención social: "La intervención socioeducativa debe entenderse, en esencia, como elemento clave de la intervención social desde el enfoque comunitario propuesto, dado su carácter procesual que, por definición, supera la tarea asistencial y de derivación, estableciendo marcos de trabajo educativos relacionales con la intención de conseguir la mayor autonomía en el sujeto, la mejora de las condiciones de vida del mismo, la adaptación a los diferentes contextos de su desarrollo y la superación de sus dificultades" (Ley 12/08 de Servicios Sociales: 31.845).

Este acercamiento a la definición de la tarea socioeducativa supone un hito en la normativa y apunta un importante avance en dos aspectos:

- La incorporación como servicio en el catálogo de prestaciones y servicios entre los de atención primaria y, por ello, de responsabilidad municipal, el Servicio de Intervención Socioeducativa y Psicosocial y Servicio de Intervención Socioeducativa y Psicosocial con Familias de competencia foral ${ }^{1}$.

${ }^{1}$ También como prestación definida en el anexo del Decreto de Cartera que debe desarrollarse en gran parte de los servicios de esta.
- La consideración profesional de los y las educadoras sociales como profesionales troncales de los servicios sociales junto con las y los trabajadores sociales: "De este modo, a las funciones propias del trabajo social de los trabajadores y trabajadoras sociales deben incorporarse las derivadas de los servicios de carácter socioeducativo ejercidas por los educadoras y educadores sociales que ya se vienen desarrollando desde hace tiempo, que, junto con los y las anteriores conformarían el cuerpo profesional básico del Sistema de Servicios" (Ley 12/o8 de Servicios Sociales: 31.845).

\section{Algunas aportaciones para la revisión de la intervención socioeducativa y psicosocial en el ámbito local para su definición y desarrollo}

Como ya se ha dicho, este artículo tiene como finalidad contribuir a la reflexión en torno al desarrollo de las actuaciones de naturaleza socioeducativa (y psicosocial) en el ámbito municipal. En primer lugar, hay que decir que estas actuaciones deben presentarse agrupadas de modo coherente en un solo servicio, el 1.3, de intervención socioeducativa y psicosocial (Decreto 185/2015) y ampliar su población destinataria. El servicio que nos ocupa, además de ser respuesta troncal en el marco de la prevención y a la desprotección infantil y adolescente (leve y moderada), ha de atender desde sus tareas propias a las personas adultas que atraviesan por situaciones de exclusión desde la atención

Como consideración general, vemos necesario entender el desarrollo de la cartera de servicios en clave de oportunidad para la revisión de los programas actuales y la progresiva implementación de mejoras y cambios en ellos. Esta revisión, supera la continuidad de las actuaciones en desarrollo "solo agrupadas" en un servicio con un único nombre y, desde la reflexión profesional, debe posibilitar el avance hacia la definición del servicio en aspectos que favorezcan, a nivel general, a:

- la conformación de un modelo consensuado de servicio de carácter municipal, adaptado a la realidad y a las necesidades de cada territorio;

- un mejor ajuste de las respuestas a las necesidades, centradas en las personas y sus procesos, unificadas, continuas y sin intermitencias;

- la mejora y diversificación de los actuales programas y la incorporación de nuevas modalidades socioeducativas y de nuevos destinatarios y colectivos;

- la recuperación de modelos relacionales y mayor calidad en las metodologías de aplicación; incorporación de nuevas modalidades y atención socioeducativa a nuevos colectivos; $y$ 
- el ajuste en la dotación de recursos para el desarrollo del servicio de índole profesional, técnica y material.

Al final de esta propuesta presentamos a través de un esquema un acercamiento al modelo de servicio que desarrolla diez grandes bloques de contenido que, aplicados, entendemos que facilitan su definición, de manera adaptada a la realidad municipal y de los servicios sociales.

De cara a facilitar la definición del servicio en cada municipio, aportamos una serie de consideraciones que ayuden a determinar diferentes elementos a revisar en cada una de las realidades de los departamentos de servicios sociales relacionados con los siguientes aspectos:

- La trayectoria de los servicios hasta el momento.

- La rigurosa respuesta a las necesidades de las personas.

- El ajuste de procedimientos adaptados de intervención.

- La diversificación de la intervención y la incorporación de nuevos destinatarios.

- Los modelos de intervención, marcos para ella y despliegue de modalidades de respuesta.

- El trabajo comunitario y el desarrollo de la comunidad.

- Las relaciones de coordinación con el resto de servicios y recursos.

- La ubicación ajustada del servicio en los servicios sociales municipales.

- La relación entre la atención primaria y la secundaria.

- La organización y la planificación del servicio.

\subsection{La trayectoria de servicios y actuaciones de intervención socioeducativa (y psicosocial)}

La mayor parte de los municipios de la Comunidad Autónoma del País Vasco ya desarrolla servicios o programas de intervención socioeducativa y/o psicosocial a través de diferentes modelos (programas de educación de calle, programas de intervención familiar, educadores-as de menores, educadores de familia etc., agrupados, como en el caso de Bizkaia, en los equipos de intervención socioeducativa y comunitaria) y puede asegurarse que estos:

- han contribuido de modo sustantivo a la diversificación de los servicios sociales desde mecanismos de observación proactiva y de detección de situaciones de dificultad;

- en general, tienen un considerable conocimiento de la realidad social del municipio y desarrollan, en muchos casos, un más que razonable trabajo comunitario, creando redes y generando iniciativas de dinamización estables de carácter interdisciplinar;

- igualmente, han desarrollado sistemas de coordinación de carácter continuo y de colaboración conjunta con el resto de servicios y recursos que operan en el municipio.

La norma que nos vincula insta al refuerzo y revalidación de lo socioeducativo, junto con el trabajo social como actividad principal. El desarrollo del Servicio de Intervención Socioeducativa y Psicosocial como servicio unificado (algunos municipios ya van incorporando, al menos la nueva nomenclatura), debería establecer una transición pausada que garantice la continuidad de los procesos socioeducativos en desarrollo y optimice la relación y coordinación ya en marcha con los diferentes agentes, recursos y servicios y con la propia comunidad.

\subsection{La respuesta ajustada a las necesidades de las personas, grupos y colectivos desde diagnósticos de consenso}

No cabe duda de que una buena intervención viene determinada por un adecuado diagnóstico, centrado en lo importante y en atención a un enfoque riguroso. En este orden de cosas, parece necesario superar paradigmas que, con cierta rigidez administrativa que acaban, demasiadas veces, respondiendo más a las necesidades del servicio que a las de las personas a las que va dirigido.

[...] proponemos una delimitación de las finalidades del servicio que, de manera progresiva, contribuyen a la definición de un determinado modelo: la respuesta ajustada a las necesidades de las personas con las que se interviene [...]; la adaptación de las personas al contexto social, cultural y de convivencia; [...] la participación real y activa de las personas para el ejercicio de la ciudadanía; [...] la adaptación de los recursos y los servicios a toda la población; [...] las transformaciones de carácter comunitario; [...] la transformación del modelo social, educativo y de convivencia, derivado del análisis desde la cuestión social, con perspectiva de equidad, en clave de justicia social e igualdad de oportunidades (Otaño, 2016: 45-46).

Es el momento, pues, de idear metodologías de diagnóstico que utilicen espacios y tiempos que permitan la observación cotidiana y el diagnóstico pausado y progresivo permitiendo atravesar las distintas etapas de análisis, desde el síntoma detectado hasta las necesidades ya definidas en sus diferentes fases:

- De observación, desde el cercano conocimiento de los y las profesionales, y su implicación, para lo cual es necesario disponer de tiempo y marcos para las interacciones y relaciones normalizadas de encuentro. 
- De identificación de dificultades, a partir de una mirada objetiva y a través de indicadores centrados en ellas.

- De valoración de la situación personal y de la adaptación de las personas al entorno y a los contextos en los que se desarrolla su vida, con la información relevante para determinar el nivel de riesgo de desprotección, de exclusión, de vulnerabilidad o de desvinculación.

- De definición de necesidades, atendiendo a las de construcción personal, de desarrollo social y de socialización, así como a las de carácter organizativo y de estructura.

Unido a esto está el diseño y la elaboración de las diferentes herramientas que han de ser elaboradas de modo consensuado, partiendo de cierta unificación de criterios entre las personas profesionales sobre el análisis de las situaciones a abordar, lenguajes comunes y procedimientos para su elaboración compartida.

\subsection{El procedimiento de intervención personalizada e interdisciplinar}

El desarrollo de todos los servicios ha de promover una respuesta a las necesidades de las personas lo más adaptada posible, para lo que se hace preciso proponer modelos definidos de atención y métodos concretos de intervención basados en esta rigurosa adaptación. En este sentido, el procedimiento para la intervención debe ser personalizado a través de un plan, en principio, en su entorno habitual y con carácter interdisciplinar que debe estar bajo la responsabilidad de un profesional de referencia.

El Cuadro 2 expone el procedimiento de intervención a seguir y a continuación se presenta una breve explicación de las distintas fases, matizando las diferentes actuaciones y tareas correspondientes a cada una de ellas.

- 1a Fase: Detección. La detección de las situaciones de dificultad, posibilitada a partir de la oferta propia del propio departamento, la atención directa con las personas en los procesos de ayuda, tanto desde las unidades de trabajo social como de los programas y servicios de intervención socioeducativa. Igualmente, otros sistemas o servicios, sobre todo el ámbito escolar o el sistema de salud, detectan situaciones de dificultad que han de ser notificadas dentro de un sistema de coordinación estable y continuo.

- 2a Fase: Recepción. La recepción de las situaciones de dificultad mediante registro como persona usuaria de los servicios sociales con la apertura de una historia social

- $3^{\text {a }}$ Fase: Investigación. La recogida de información adecuada y rigurosa, que viene de los análisis de la situación tanto desde la observación directa, en los marcos de intervención socioeducativos (medio abierto, marcos grupales) conformados, como desde las entrevistas para la identificación de las dificultades. También son valiosas las aportaciones de profesionales de otros servicios.

- 4a Fase: Valoración y orientación. La fase de valoración determina las necesidades a partir de un análisis riguroso de las personas, familias y colectivos, clasificándose la situación según el riesgo de desprotección o exclusión y articulándose la orientación debida a la elaboración del plan de intervención municipal o derivando la situación a la Administración foral.

- 5a Fase: Elaboración y desarrollo del plan de intervención. El equipo de profesionales responsable del Servicio de Intervención Socioeducativa y Psicosocial diseña el plan de intervención definiendo las necesidades y formulando objetivos medibles y sujetos a plazos, la asignación de modalidad y la programación de la propia intervención y su desarrollo. Asimismo, se estructura un sistema de coordinación que posibilita el seguimiento de la intervención, el flujo continuo de información relevante y el contaste de los logros o dificultades que se van presentando, y se establecen las vías de colaboración coordinada con otros agentes, recursos y servicios.

- 6a Fase: Evaluación. La evaluación es la fase final del proceso con momentos para su revisión intermedia. Establece el alcance de la consecución de los objetivos perseguidos, la respuesta a las necesidades, de la superación de dificultades así como en la adquisición por parte del sujeto de los recursos personales a proyectar en el resto de contextos en que se desarrolla su vida. Por último, permite el cierre de la intervención, o, en su caso, establecer las orientaciones futuras, ya sea la continuidad desde otra modalidad de respuesta o la necesidad de derivarla hacia instancias forales.

Como señalamos en el resto de la propuesta de este artículo, estamos ante la oportunidad de revisar los diferentes modos con los que cada departamento de servicios sociales desarrolla la intervención, desde la detección hasta el cierre, con la finalidad de establecer, a partir de la exigencia de las instancias del Gobierno vasco, un proceso de intervención adaptado propio definido que:

- delimite los contenidos y las tareas a desarrollar en cada fase, con el fin de optimizar y dedicar el tiempo justo y necesario para cada fase a partir de la información significativa, sin alargar innecesariamente alguna de ellas;

- favorezca la participación de todas las personas profesionales con responsabilidades en la intervención, tanto directa como indirecta y en todas las fases del procedimiento;

- defina, de una parte, la utilización adecuada de los instrumentos propuestos desde la 


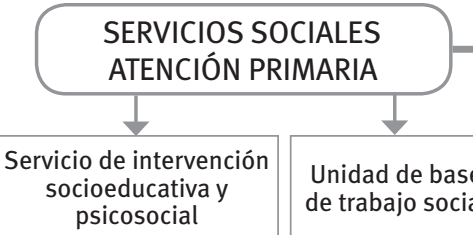

Otros recursos y servicios del sistema

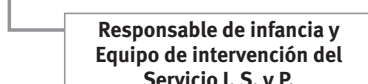
Servicio I. S. y P.
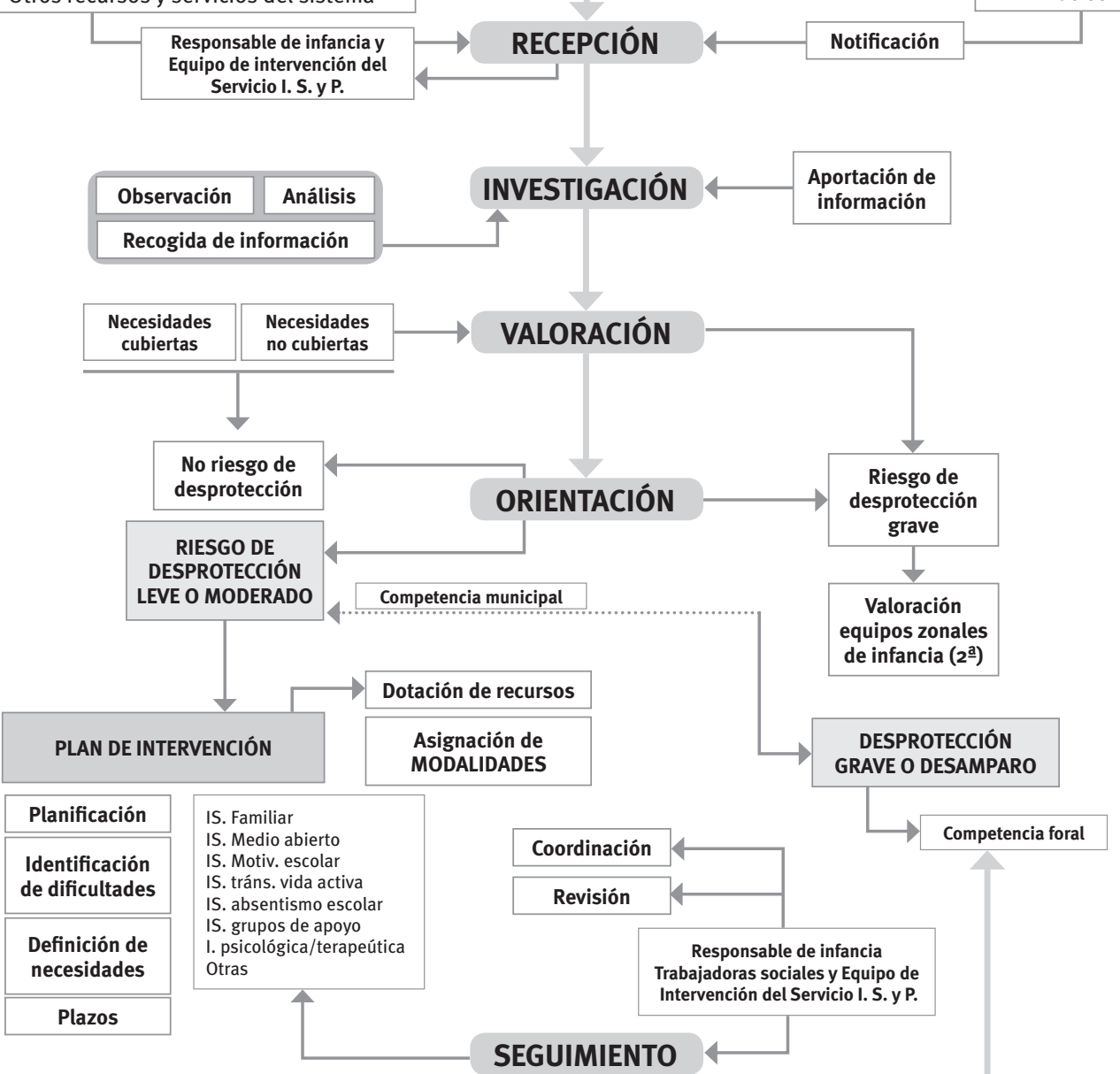

Responsable de infancia Trabajadoras sociales y Equipo de Intervención del Servicio I. S. y P.

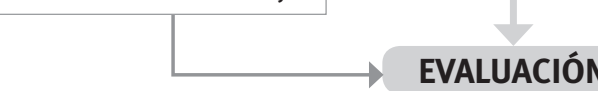

\begin{tabular}{|c|c|}
\hline CONTINUIDAD & Consecución de objetivos \\
\hline Prórroga \\
\hline Plan de cierre \\
\hline Plazo corto \\
\hline
\end{tabular}

Nueva valoración

\section{CIERRE}


dirección de servicios sociales de la $\mathrm{CAPV}^{2}$ en las fases iniciales previas al plan de intervención propiamente dicho y su uso ajustado a su finalidad;

- la elaboración de herramientas necesarias para la derivación, detección, diagnóstico de necesidades y el plan de intervención como tal; y

- el diseño de una ficha compartida por todos los y las profesionales y que pueda ser global de todo el procedimiento y en todas sus fases parece recomendable.

Se aprecia la necesidad de una herramienta común para optimizar los procesos de intervención con las personas en el servicio de información, valoración, diagnóstico y orientación. En lo que se refiere a los procedimientos de intervención en el ámbito de los servicios sociales de atención primaria, se ha puesto claramente de manifiesto la necesidad de avanzar en el desarrollo de herramientas comunes de intervención y en la mejora de su uso (Ararteko, 2016: 166).

\subsection{Las personas adultas en situación de exclusión y la respuesta desde el servicio}

Los servicios municipales de naturaleza socioeducativa y psicosocial han estado vinculados hasta ahora, por tradición y por razones de cumplimiento legal, con la respuesta al colectivo de niños y niñas y adolescentes, y sus familias, principalmente, en las situaciones de desprotección leve y moderada, aunque también en otras de mayor gravedad sin una valoración específica en ese sentido.

Un análisis pormenorizado de la realidad del territorio nos conduce a la detección de situaciones de personas y colectivos con evidentes necesidades de adquisición de recursos personales y sociales y de acompañamiento socioeducativo. Nos referimos, fundamentalmente, a personas adultas, dentro o fuera de sus marcos familiares, en situación de dificultad y, en muchos casos, en exclusión; muchas veces, aisladas, desvinculadas o con dificultades de muy diversa naturaleza y necesidades de claro abordaje desde lo socioeducativo a los que los modelos actuales, a menudo vinculados a prestaciones económicas o al empleo, no han permitido responder.

[...] no hay claros indicadores para señalar dónde situar la situación de exclusión, sin embargo, [...] las personas podemos estar "dentro" (integración social), en el límite (precariedad, riesgo, vulnerabilidad), o "fuera" (exclusión social). [...] La escasez o inexistencia de ingresos económicos, la carencia de vivienda digna, el desempleo o el empleo precario son variables objetivas [...], pero, también, las enfermedades, las dependencias y

2 Ficha Social e Instrumento de Diagnóstico Social (Decreto $353 / 13$ ), Instrumento Balora (Decretos 230/11 y 152/17), e Instrumento para Valoración de la Exclusión Social (Decreto 385/13). las deficientes relaciones sociales [...] generan aislamiento, estigmatización y, [...] discriminación social (Otaño, 2012).

El servicio que nos ocupa (1.3) no limita la edad de las personas a las que debe ir dirigido, por lo que es el momento de contemplar a las de este colectivo adulto como sujetos de intervención desde nuestra tarea. Las respuestas definidas han de materializarse en los siguientes elementos:

- Itinerarios que atraviesen todas las fases del procedimiento, tal y como se señala en apartado anterior, para lo que será necesario establecer marcos de observación y detección en el medio abierto y con el resto de recursos y servicios.

- Asignación de modalidades de carácter general ajustadas y otras específicas de respuesta de índole socioeducativa y de acompañamiento; la finalidad es, en este caso, la adquisición de la motivación y el empoderamiento que permita alcanzar la autonomía necesaria para su madurez personal y su desenvolvimiento social.

- Generación de marcos individuales, grupales, familiares o en el medio abierto, de los que se apunta un mayor detalle en la aportación siguiente.

Igualmente, es momento oportuno para, tras una rigurosa reflexión del alcance de las actuaciones y programas en vigor que tienen como centro los colectivos tradicionales, proponer respuestas de carácter mixto y dotarlas de mayor flexibilidad, o, en su caso, apostar por el diseño y desarrollo de nuevas modalidades de respuesta que incorporen a las personas hasta ahora insuficientemente atendidas.

En todo caso, me parece importante subrayar que acotar la finalidad del SVSS [sistema vasco de servicios sociales] a la integración social, por un lado, y orientar su acción a toda la población, por otro, no ha de suponer perder de vista: ni la orientación transformadora y emancipadora de la intervención [...] y a su inclusión social con la máxima autonomía posible, en cooperación con otros recursos y sistemas; ni la perspectiva de las personas, familias, colectivos y comunidades que afrontan mayores dificultades para la integración social (López-Arostegi, 2016: 4).

Resulta necesario dar un nuevo impulso al Servicio de Intervención Socioeducativa y Psicosocial para que extienda su actuación a: 1) en línea con lo dispuesto en el Decreto de Cartera, personas adultas con necesidad de apoyos socioeducativos o psicosociales de baja intensidad, esto es, personas en riesgo de dependencia o familias y personas en riesgo de exclusión (falta de apoyos sociales, soledad, aislamiento...) (Ararteko, 2016: 176).

La necesidad de impulsar la intervención socioeducativa y psicosocial, más allá de 
su prestación en centros o equipamientos específicos. [...] La potencialidad de las intervenciones en el medio en el que se encuentran las personas en situación de exclusión, con los recursos comunitarios disponibles, habría de permitir por un lado mantener en su entorno a aquellas personas usuarias para las que es beneficioso dicho entorno y supone un sostén para su proceso de inclusión (Larrion, Leturia, Zalakain y Zabaleta, 2019: 58).

Previa a la asignación de la modalidad, ya hemos señalado la importancia de ajustar las herramientas que para la valoración deban usarse de forma que permitan delimitar el grado de desprotección o exclusión en el caso de las personas adultas. Esta consideración tiene en cuenta el reconocimiento de que en muchas de las situaciones a abordar las personas que componen el colectivo que puede estar atravesando dificultades de inclusión ya están siendo atendidas desde modalidades básicas, sobre todo de intervención familiar, aunque no con la especificidad que requiere su situación. En cualquier caso, la respuesta va a venir favorecida por el hecho de ser integrada, de un modo sistematizado y a partir de diagnósticos serios.

\subsection{Los marcos para la intervención y las modalidades de respuesta ajustadas}

Vamos a referirnos a continuación a las mejoras oportunas que en atención directa y con la persona como centro de la intervención deben darse. Se trata de preservar modelos y metodologías que:

- generan marcos relacionales de intervención a partir del desarrollo de relaciones personalizadas, basadas en la horizontalidad y en el cercano conocimiento y el reconocimiento del otro como alguien "único", conformando interacciones positivas y de confianza que permitan alcanzar la vinculación para orientar a las personas en sus opciones hacia la mejora de su situación;

- posibilitan procesos educativos que exigen tiempo de dedicación necesario y suficiente, favorecidas por el servicio, materializados en los planes de intervención; y

- responden a una intencionalidad de cambio dirigida a la rigurosa respuesta a las necesidades de las personas usuarias a partir de la adquisición de recursos personales que los posibiliten.

La definición del servicio en su ficha de la cartera apunta el despliegue de diferentes modalidades que conformarían el servicio. Para ello, parece necesario hacer una propuesta que, adaptada por cada municipio según su necesidad, establezca cuáles son esos marcos educativos de intervención, entendidos estos como los espacios y formatos que se desarrollarían en las distintas modalidades que también proponemos. La reflexión técnica y la experiencia en la tarea socioeducativa directa y en la de seguimiento y apoyo a numerosos equipos de intervención nos lleva a establecer como marcos para ella los que señalamos a continuación:

- Marcos de intervención familiar, desarrollados en sesiones familiares (sobre todo, en el domicilio) con todos y cada uno de sus miembros.

- Marcos de intervención grupal, concebidos como posibilitadores, incluyentes y adaptados a todas las personas.

- Marcos de intervención individual, como complemento de las propuestas grupales y familiares, también en atención al seguimiento y revisión de procesos y acompañamiento personal.

- Marcos de intervención en medio abierto, espacios naturales de convivencia y encuentro inicialmente "de otros", haciéndolos comunes; facilitan la detección de situaciones de dificultad y el acercamiento natural a las personas, permitiendo el análisis continuado del territorio y el exhaustivo conocimiento de la comunidad y de su dinámica

Entendidos estos marcos como básicos, van a suponer el modelo metodológico en la atención directa que serán desarrollados en las diferentes modalidades posibles que, como orientación, se apuntan en el Cuadro 3.

\begin{tabular}{|l|}
\hline Cuadro 3. Propuesta de modalidades a desplegar desde el \\
Servicio de Intervención Socioeducativa y Psicosocial \\
\hline \begin{tabular}{l} 
Modalidades de carácter general \\
$\rightarrow$ responden a situaciones de dificultad multifactorial \\
\hline - Intervención socioeducativa familiar. \\
- Intervención socioeducativa en medio abierto. \\
- Intervención socioeducativa para la motivación escolar. \\
- Intervención socioeducativa con personas adultas. \\
\hline $\begin{array}{l}\text { Modalidades de carácter específico } \\
\rightarrow \text { pretenden dar respuesta a personas y colectivos desde una } \\
\text { dificultad objetiva más concreta. }\end{array}$ \\
\hline - Intervención socioeducativa para el tránsito a la vida activa. \\
- Intervención socioeducativa ante el absentismo escolar. \\
- Intervención en grupos de apoyo a colectivos (adultos/as con \\
dificultades o en exclusión).
\end{tabular} \\
- Intervención psicológica individual o familiar. \\
\hline $\begin{array}{l}\text { Modalidades de carácter comunitario } \\
\rightarrow \text { centra su atención en la comunidad en general como sujeto. }\end{array}$ \\
\hline - Intervención comunitaria.
\end{tabular}

Fuente: Elaboración propia.

El desarrollo del servicio que nos ocupa va a ser muy singular en el caso de los pueblos pequeños dadas las características de sus departamentos, los bajos recursos de los que disponen y la estructura de "supervivencia" en los que sus profesionales resuelven la respuesta a las necesidades de las personas. Nos estamos refiriendo a municipios que en su mayoría no superan los 1.000 habitantes que, en muchos casos, tienen sus servicios sociales mancomunados, con profesionales compartidos $y$, 
la mayor parte de las veces, solo una profesional del trabajo social. En este caso, no podemos pensar en un despliegue de modalidades muy diversificado, pero sí un servicio que, si bien compartido entre varios municipios, permita la respuesta lo más ajustada posible a las personas del territorio mediante un modelo más específico.

En algunos casos, igualmente, se podrían establecer a partir de acuerdos imaginativos, ramificaciones de servicios implementados en municipios más grandes y con carácter referente en la comarca para garantizar la cobertura de la atención en pueblos más pequeños de su radio de influencia.

\subsection{La dimensión y el trabajo comunitario: enfoque, perspectiva y desarrollo comunitario}

Es preocupante que en el último tiempo la dimensión comunitaria haya quedado relegada a un plano poco relevante en las tareas desarrolladas por los servicios sociales y por los programas y servicios de intervención socioeducativa y, en algunos casos, incluso eliminada de ellos. Es por esto por lo que debemos recordar que la intervención socioeducativa y psicosocial, tanto en la concreción de sus marcos como desde su propia naturaleza, ha de ser comunitaria, anclando esto, desde nuestro punto de vista, en cuatro grandes razones que atienden:

a. de una parte, a una base antropológica -la vida de las personas deviene de su desarrollo en vinculación con el entorno-; así, es preciso favorecer esta conexión de adaptación y adaptabilidad;

b. de otra, a lo social, lo económico y lo cultural -las personas, los colectivos y las comunidades se desarrollan en realidades sociales y culturales con características diferenciadas y modelos económicos y de convivencia específicos-;

c. lo estrictamente socioeducativo -la adquisición de recursos personales para el desarrollo individual y social, el ajuste entre las personas y los contextos en los que se desarrolla su vida y el de estos contextos como facilitadores de respuesta a las distintas situaciones-; $y$, finalmente,

d. a la legislación -la Ley 12/o8, en sus principios, establece en todos los servicios y prestaciones la troncalidad del enfoque comunitario y el compromiso de aplicación de lo comunitario como perspectiva y modelo)-.

Es por esto por lo que la comunidad también tiene que entenderse como sujeto de la intervención y su desarrollo es también finalidad del servicio, dado que lo comunitario posibilita la promoción de cambios de índole social orientados a la transformación del marco de convivencia en clave de inclusión y de pertenencia, para lo cual deben adquirir especial relevancia las Administraciones Públicas y, dentro de ellas, el servicio que nos ocupa.
[...] podemos decir que la comunidad es el territorio y todas las personas que habitan en él, pero también las agrupaciones y recursos que, desde la iniciativa social, operan en el mismo, y los servicios públicos con responsabilidad y, sobre todo, las redes y relaciones que se generan entre ellas (personas, agrupaciones, recursos, servicios) (Otaño, 2016: 50).

Pero hablar de trabajo comunitario implica fijar bases para lo que se puede entender como su finalidad misma, el crecimiento mismo de la comunidad y el desarrollo comunitario en definitiva y, en este sentido, la contribución por parte del servicio y de sus profesionales se materializa en diferentes actuaciones que, agrupadas, permiten un proceso en distintas fases:

- las primeras, relacionadas con la territorialidad, es decir, presencia, disponibilidad y conocimiento de lo que pasa en el territorio;

- después, actuaciones relacionadas con la participación en la vida comunitaria y en las iniciativas que se desarrollan, que posibiliten la adquisición de protagonismo y significación dentro de ella; $y$, por último,

- la dinamización comunitaria y el trabajo en red, promoviendo la cultura participativa, el respeto a la diversidad y el diálogo.

[...] la Red comunitaria puede definirse como la unión formal de varias personas y agentes responsables de recursos y servicios, el nivel de relaciones que se genera en esta unión, su proceso en relación a los contenidos que se trabajan y los proyectos e iniciativas que derivan de estos mismos procesos como tarea troncal del servicio desde su naturaleza, su sentido y su finalidad (Otaño, 2016: 51).

\subsection{La corresponsabilidad de las intervenciones con el resto de recursos y servicios del territorio}

El fin de la intervención socioeducativa tiene que ver con la adaptación de las personas a los diferentes contextos en los que se desarrolla su vida, pero también la adaptación (adaptabilidad) de estos y sus respuestas a cada una de ellas. En este sentido, el desarrollo del servicio debe garantizar la mínima corresponsabilidad respecto a la intervención a través de la coordinación con el resto de recursos y servicios que, por su naturaleza o destinatarios, tengan carácter preferente, o sean especialmente significativos.

Para la identificación de los recursos y servicios con los que se debe establecer una relación coordinada, es necesario un estudio sistemático de los diferentes agentes que operan en el territorio que permita determinar el alcance de sus intervenciones, sus destinatarios principales y las posibilidades de trabajo conjunto que propicie respuestas integradas. Una vez priorizados los agentes, recursos y servicios, 
son tres los principales momentos en los que se hace decisiva la coordinación:

- A la hora de la detección, haciendo fundamental el trabajo compartido a través de sistemas de coordinación establecidos, con lenguajes identificados y orientaciones comunes suele ser más eficaz que la mera puesta en marcha de protocolos de derivación.

- Para el desarrollo de los planes de intervención, ya que la coordinación permite la orientación común, las tareas complementarias y el contraste constante.

- En el desarrollo del espacio comunitario, es claro que los marcos de coordinación hacen posible la conformación de redes.

El servicio asume un carácter proactivo y dinamizador de la coordinación, identificando al resto de agentes como copartícipes en las intervenciones y definiendo un proyecto de coordinación con cada uno de ellos. Este proyecto de coordinación se sustancia a partir de sistemas de coordinación, que, definidos en contenidos y tiempos y mediante procesos, posibiliten las orientaciones comunes que respondan a las personas usuarias en una misma dirección y, a su vez, permitan reconocer la proyección de sus logros y cambios en otros espacios.

\subsection{La ubicación funcional del servicio en el marco de un único sistema de servicios sociales}

El desarrollo del servicio en atención al mandato de la legislación vigente requiere de una ubicación que garantice la conexión del servicio en la acción general del departamento de servicios sociales municipal y su funcionalidad, así como de su ajuste a partir de dos elementos fundamentales:

- la claridad de los procedimientos para la atención, y

- la definición de funciones que son responsabilidad de los profesionales de cara al desarrollo del servicio

Parece pertinente en este momento, tal como ya se ha dicho anteriormente, repensar el contenido, las tareas y las herramientas para la ejecución de cada una de las fases del procedimiento desde la detección, el diseño del plan de Intervención y el cierre o derivación de los procesos. Es preciso repartir la tareas de modo coherente, delimitando el papel de los distintos profesionales en el procedimiento y en cada una de sus fases, no dejando a ninguno de ellos o ellas relegados a informantes o simples ejecutores, sino haciendo a todos y todas las profesionales responsables y partícipes de todas ellas.

Así, la función principal de los y las educadoras sociales está en la atención directa y en la generación de marcos y espacios educativos para el desarrollo de procesos de intervención, su activación y liderazgo en ellos, siendo el desarrollo de espacios e intervenciones de carácter psicológico o terapéutico responsabilidad de los y las profesionales de la psicología. A estas funciones han de unirse las de carácter organizativo, diseño y de coordinación, ya comentadas con anterioridad. Por su parte, también la buena ubicación física del servicio y de sus profesionales contribuye a una situación más adecuada en el modelo organizativo general. La disposición del espacio de trabajo en el mismo departamento que el resto de profesionales de los servicios sociales, con lugares para tareas y materiales comunes, etc., facilita claramente la integración, la comodidad y la eficacia del equipo.

\subsection{El tránsito de procesos de intervención entre la atención primaria y la atención secundaria}

En esta concepción de los servicios sociales como un único sistema en el que cada Administración desarrolla las funciones e intervenciones que son de su competencia, conviene señalar la importancia del cuidado en los procesos de transición de las intervenciones de la atención primaria a la secundaria que, en muchos casos, es motivo de preocupación y de insatisfacción por parte de muchos de los y las profesionales tanto en el ámbito local como el territorial. Esta concepción de sistema coherente debería proyectarse a los de protección e inclusión, yendo así desde la prevención de las situaciones de dificultad hasta el abordaje de la extrema gravedad, pudiendo hablarse de un único sistema de protección y uno de inclusión aún desde las competencias de cada Administración. Concretar una buena transición entre ambos niveles del sistema no siempre es tarea fácil dado que, en muchos casos, las situaciones que se han de abordar y la a menudo rigidez de los procedimientos y de las instituciones no ayuda.

En la línea de aportar algunas ideas que favorezcan el ajuste podemos decir que ambas instituciones, situando a la persona y su proceso en el centro, pueden garantizar una atención global, integral y no fragmentada a partir de contar con dispositivos y recursos para una rápida respuesta a las distintas situaciones. De otro modo, si centralizamos la atención en la tarea de la Administración, se produce un debilitamiento de la unidad de acción como respuesta a los derechos de las personas.

En este orden de cosas, el conocimiento concreto por parte de todos los y las profesionales del sistema de los distintos equipos y servicios de cada una de las Administraciones, de los procedimientos y las estructuras existentes en cada institución, favorece la coherencia y preserva el carácter continuo de las intervenciones. Esto hace necesario:

- definir y sistematizar una coordinación que facilite el traspaso de información de modo fluido y constante, cuidando la continuidad de 
los procesos e itinerarios de las personas, sin intermitencias ni ruptura de procesos;

- poner en marcha mecanismos que reconocen el conocimiento exhaustivo y cercano por parte de la atención primaria de las situaciones de las personas, teniendo en cuenta que es la persona atendida la que las protagoniza, en su recorrido, muchas veces con fuerte vinculación con los o las profesionales; y

- asegurar la permanencia de un o una profesional de referencia a lo largo de las distintas intervenciones desde una planificación más coherente y una mejor orientación a las personas en procesos de intervención.

\subsection{La organización del equipo para el desarrollo del servicio y el sistema la planificación}

La organización del servicio y de sus profesionales es requisito para garantizar su eficacia, la ubicación coherente anteriormente señalada y la calidad de las intervenciones. Centrados en la persona responsable orgánica del departamento de servicios sociales, apuntamos a continuación una serie de actuaciones que esta debe desarrollar o, en su defecto, delegar, estableciéndose como funciones propias de la organización del servicio:

- Impulso a la implementación y desarrollo de un sistema de planificación transversal y estratégico del servicio que nos ocupa. Este sistema responde a las necesidades derivadas de un riguroso análisis y tiene carácter global, incluyendo sus elementos básicos (fundamentación, objetivos, acciones y evaluación), respeta los procesos y garantiza su efectividad midiendo el alcance de las actuaciones y evaluando sus logros.

- Liderazgo en la organización del servicio a través de un seguimiento eficaz y con suficiente conocimiento. De este modo, se asume la responsabilidad de las respuestas que desde el departamento se establecen.

- Aportación de los apoyos humanos y técnicos necesarios, así como la dotación de los recursos materiales que los profesionales han de disponer para el desarrollo de su tarea, tanto en la atención directa como en la indirecta.

- Coordinación interna en un sistema definido; es decir, las funciones, la asignación de tareas a cada profesional y la coordinación interna entre ellos en una sistemática de reuniones del equipo de intervención. Este equipo está conformado por los profesionales responsables de la atención directa (educadores/as sociales y psicólogos/as), la persona coordinadora del equipo y las/os trabajadoras/es sociales de base con atribuciones en la intervención

- Promoción de la coordinación con otros sistemas de servicios desde la representatividad de los servicios sociales en la puesta en marcha de las relaciones coordinadas comentadas anteriormente.
- Fomento del trabajo comunitario y el desarrollo de la comunidad, manteniendo en todo momento la reflexión de la dimensión y el enfoque comunitario de la acción social y la atención respecto a la importancia de la comunidad y la contribución de los servicios sociales a su desarrollo.

En todos los casos, tanto educadores y educadoras sociales como psicólogos y psicólogas participan y tienen funciones definidas en la planificación del servicio, en el diseño de los planes y proyectos de intervención, en la coordinación con otros y en la acción comunitaria.

Ni que decir tiene que un claro objetivo de la organización es que las personas profesionales disfruten de la mayor seguridad posible, lo cual revierte en el fomento de su motivación y en el nivel de exigencia con las intervenciones a desarrollar. Relacionado con esto, respecto a las personas encargadas de la ejecución del servicio, cabe señalar la importancia de tres aspectos que tienen, a nuestro entender, especial relevancia y que, a su vez, implican a la entidad que los desarrolla:

- La conformación de equipos integrales de intervención formados por los y las educadoras sociales para el desarrollo de la intervención socioeducativa y las personas profesionales de la psicología para las de naturaleza psicosocial.

- La estabilidad del equipo de intervención, favorecida por las buenas condiciones de contratación sujetas al convenio de intervención social del territorio histórico correspondiente; en este orden de cosas, hay que decir que se deberían ya superar las contrataciones, que en algunos casos aún se siguen ejecutando, de intervenciones por horas y en condiciones de alta precariedad. Las personas profesionales deberían disfrutar de un marco contractual que garantice la incorporación a un equipo con los requisitos que aquí se comentan.

- La incorporación en este equipo de un o una profesional con funciones de coordinación técnica que, centrado en el apoyo a los profesionales de la intervención, contribuye a la calidad del servicio, estableciendo procesos de coordinación de carácter organizativo y de acompañamiento técnico, esto es, el seguimiento y asesoramiento técnico, la capacitación y el soporte emocional necesario en servicios de esta naturaleza.

\section{Acompañamiento técnico a los municipios para el desarrollo del servicio que marca la cartera}

En estos momentos, los diferentes departamentos municipales están incorporando, si no lo han hecho ya, en mayor o menor medida y con más o menos dificultades, los cambios necesarios para adaptar sus actuaciones a los servicios que la cartera exige en el 
ámbito municipal. El paso al nuevo planteamiento más integrado, en muchos casos, se presenta con cierta complejidad debido a las diferentes dinámicas internas de cada departamento y a las estructuras existentes, la falta de tiempo para "pararse" a reflexionar y definir cambios, las inercias de los modelos de atención a la infancia y adolescencia en vigor o la necesidad de la atención de nuevos destinatarios hasta ahora no contemplados desde servicios de esta naturaleza. A continuación, queremos apuntar un pequeño acercamiento hacia las actuaciones que, con carácter facilitador, puede desarrollarse para el paso de los programas y servicios de naturaleza socioeducativa y psicosocial a la conformación de un servicio integral e integrado.

Con carácter piloto, con la finalidad de generar procesos de asesoramiento técnico para el tránsito al nuevo servicio, queremos citar la experiencia desarrollada ${ }^{3}$ con este cometido con los departamentos de servicios sociales de seis municipios de Gipuzkoa'; se trató de un proceso fundamentalmente educativo del que apuntamos sus características en el Cuadro 4.

\section{Cuadro 4. Características de los procesos de asesoramiento técnico para el tránsito al Servicio de Intervención Socioeducativa y Psicosocial}

- Parte del reconocimiento de las trayectorias de servicios y programas que se han dado hasta el momento en el municipio, así como los equipos y los profesionales que los han llevado a cabo.

- Se desarrolla a partir del grupo de trabajo conformado por las personas profesionales del equipo con la persona asesora que acompaña el proceso, en sesiones de asesoramiento técnico en el lugar de trabajo del equipo

- Establece dos tiempos: uno para la definición de necesidades y el diseño de las actuaciones de respuesta, y otro con la propia implementación de estas actuaciones para garantizar el nuevo alcance del servicio.

- Consta de tres instrumentos fundamentales: por un lado, la idea a transmitir, básicamente, el modelo de referencia de servicio, del que presentamos un esquema y sus principales elementos en el apartado siguiente; una persona asesora técnica cuya función es diseñar el proceso, tutorizar, pautar, acompañar y asesorar en el mismo desde la pauta y orientación; y, por último, diferentes herramientas diseñadas ad hoc para las distintas fases del tránsito que permitan el análisis de necesidades, la reflexión y dar contenido a las actuaciones a implementar.

Fuente: Elaboración propia.

[...] un equipo de trabajo conformado por los y las distintas profesionales de los servicios sociales, las-os trabajadoras-es y educadoras-es sociales, principalmente, en un mismo nivel de reflexión y de aporte; [...] cruzar las preocupaciones de cada uno de los perfiles profesionales para centrar el contenido del servicio en su definición [...] favorece una experiencia que, por una parte,

${ }^{3}$ Equipo conformado por Ainhoa Martín Blázquez, Fermín Martín Álvarez y Jesús Otaño, educadores/as sociales/asesores/as técnicos/as. Bajo la cobertura del Consorcio para la Educación Compensatoria y la Formación Ocupacional y la Diputación Foral de Gipuzkoa. 2018). facilita un mejor conocimiento de las tareas y responsabilidades de cada profesional, su contraste [...], mejora las relaciones entre los diferentes profesionales desde sus perfiles, contribuye al aprendizaje común [...] incorporar una cultura de coordinación y colaboración que introduzca cambios para una mayor eficacia y corresponsabilidad [...] (Martín Álvarez, Martín Blázquez y Otaño, 2018).

En segundo lugar, derivado del anterior, en la actualidad desde el Servicio de Protección a la Infancia y la Adolescencia ${ }^{5}$ del Departamento de Políticas Sociales de la Diputación Foral de Gipuzkoa, se pone en marcha el Programa Hurbildu de acompañamiento técnico para la mejora de las actuaciones de naturaleza socioeducativa y psicosocial y la definición del Servicio (1.3) en los ayuntamientos. La responsabilidad del programa es de las personas del equipo asesor señalado anteriormente. De modo general, las características del programa son las siguientes:

- Dentro de la concepción de un único sistema de servicios sociales, pretende ser un apoyo con la finalidad de contribuir a la mejora en el desarrollo de los servicios de intervención socioeducativa y psicosocial, la prevención y el desarrollo local y comunitario.

- Está dirigido a todos los municipios de Gipuzkoa y a las personas profesionales que desarrollan los servicios sociales de atención primaria: trabajadoras/es sociales, educadores/as sociales, psicólogas/os y otras personas técnicas (técnicas/os de prevención, inserción, inmigración, etc.), así como a sus responsables técnicos y políticos.

- La oferta se materializa en la generación de marcos de capacitación a través de:

- Procesos de asesoramiento técnico, de carácter relacional, participativo y continuo desarrollados a partir de la agrupación de diferentes municipios o figuras profesionales.

- Acciones formativas dentro de una oferta general a través de diferentes formatos según su contenido y participantes (exposiciones generales, seminarios o talleres).

- La responsabilidad es de un equipo de asesores/ as técnicos/as, educadores/as sociales con amplia experiencia en la intervención y el asesoramiento en servicios sociales.

- Partimos de un modelo técnico de servicio como referencia, del que presentamos un esquema y sus principales elementos en el apartado siguiente.

Como ya se ha dicho, la materialización de los servicios de la cartera, en muchos casos, viene dificultada por la ausencia de marcos de reflexión

\footnotetext{
${ }_{5}^{5}$ Su jefe del servicio es Patxi Agiriano.
} 
en los departamentos y las dinámicas de trabajo y la abultada tarea de cada una de las personas profesionales. Experiencias de asesoramiento técnico de carácter facilitador como las que se desarrollan en Gipuzkoa podrían ser proyectadas para el desarrollo de la gran mayoría de servicios y, de este modo, realmente poder hablar de aprovechar la oportunidad para la revisión de las actuaciones de los servicios sociales en clave de mejora.

\section{Un modelo de referencia para el Servicio de Intervención Socioeducativa y Psicosocial}

En la línea de contribuir, desde el compromiso y la experiencia de años en la intervención socioeducativa, a la definición de modelos técnicos centrados en las personas y sus necesidades, con perspectiva comunitaria y de servicio público, presentamos un esquema que apunta a un modelo de referencia que, a través del desarrollo de diez grandes bloques de contenido, facilita el diseño del Servicio de Intervención Socioeducativa y Psicosocial adaptado en tres áreas diferentes:
- La fundamentación del servicio, la reflexión de la cuestión social el encargo y la derivada del análisis de las necesidades y de la experiencia práctica.

- La atención directa, relacionada directamente con la intervención con las personas, los marcos para la misma, las modalidades y actuaciones a desarrollar, así como la intervención comunitaria, entendiendo a la comunidad como sujeto de intervención.

- La atención indirecta para aspectos de coordinación, organizativos y de planificación, es decir, la gestión del servicio propiamente dicha.

Este modelo está explicado en profundidad en un documento elaborado por el autor de este articulo bajo la contratación del Consorcio para la Educación Compensatoria de Gipuzkoa, ya extinto, y la Diputación Foral de Gipuzkoa, y será presentado próximamente a los diferentes departamentos de servicios sociales en Gipuzkoa en el marco del desarrollo del programa Hurbildu, ya señalado en el apartado anterior, del Servicio de Protección a la Infancia y la Adolescencia

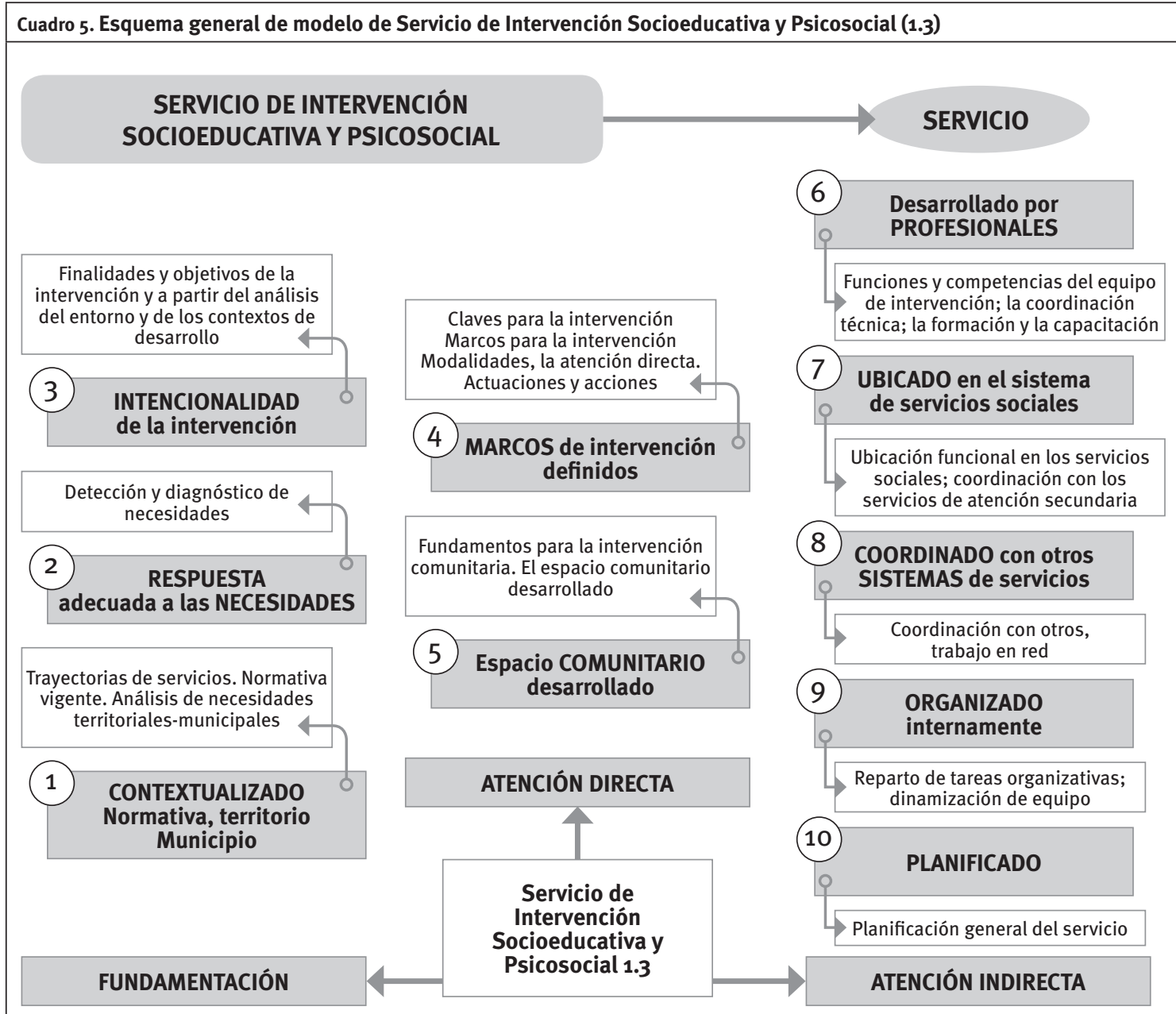

Fuente: Elaboración propia. 
Cuadro 6. Modelo de Servicio de Intervención Socioeducativa y Psicosocial (1.3); elementos que conforman los bloques de contenido

Contextualización - normativa; municipio

Conocimiento ajustado del territorio y del municipio.

Conocimiento de los recursos y servicios que intervienen u operan en el territorio-municipio.

Trabajo coordinado con otros sistemas de servicios.

Respuesta ajustada a necesidades

Procedimientos acordados de detección de situaciones de dificultad.

Marcos para la observación y la detección.

Procedimientos de detección derivación desde otros sistemas de servicios.

Identificación de dificultades.

Valoración de las situaciones en relación con los contextos de desarrollo.

Diagnóstico de necesidades clasificadas según criterios.

Herramientas para el proceso de diagnóstico de necesidades.

Intencionalidad de la intervención

Respuestas centradas en las personas como sujeto de intervención.

Respuestas ajustadas a las necesidades definidas en diagnóstico.

Respuestas dirigidas a la adaptación de los contextos de desarrollo, tanto de carácter intencional como no intencional.

Respuestas enmarcadas en la atención integral e integradora.

Definición de objetivos de la intervención en relación a la finalidad última de la intervención.

Marcos de intervención definidos

Intervención relacional.

Intervención individualizada.

Marcos de intervención ajustados a necesidades.

Intervenciones familiares desarrolladas, intervención con todos los miembros en grupos familiares, intervención en el domicilio.

Intervención en marcos grupales.

Intervención en marcos individuales.

Utilización del medio abierto como espacio de intervención, presencia de profesionales de la intervención en el medio abierto.

Desarrollo de modalidades: medio abierto, intervención familiar, intervención terapéutica, grupos de apoyo, otros.

Programación de acciones definidas y estructuradas, en respuesta a las necesidades.

\section{Dimensión comunitaria desarrollada}

Intervenciones desarrolladas desde el enfoque comunitario

Proyectos de carácter comunitario

Proyectos de desarrollo comunitario

Participación en iniciativas comunitarias

Redes generadas para el desarrollo comunitario

Desarrollado por profesionales

Equipos de Intervención interdisciplinar (educadores/as sociales, psicólogos/as y/o trabajadoras sociales).

Definición de funciones de los/as profesionales de atención directa.

Funciones propias de atención indirecta.

Competencias de los profesionales relacionadas con el saber, saber hacer, saber estar, saber ser.

Definición de funciones del coordinador: organizativas y de acompañamiento.

Formación y capacitación técnica al equipo adecuada. Formación en formatos diferentes. Formación interna o externa.

Ubicación en el sistema de servicios sociales

Competencia y responsabilidad municipal desde el departamento de servicios sociales.

Procedimiento de actuación definido en sus fases desde el departamento. Participación del equipo de intervención en las fases del

procedimiento.

Coordinación con los profesionales de servicios sociales con contenidos y tiempos definidos.

Dotación de recursos materiales para la atención directa e indirecta (espacios, económicos, tecnológicos, etc.).

Integración física y funcional del servicio en el departamento.

Liderazgo desde el departamento de servicios sociales en el desarrollo del servicio.

Relación coordinada definida entre atención primaria y secundaria.

Coordinación con otros sistemas de servicios

Coordinación con otros sistemas de servicios: para la detección y derivación; para el desarrollo de planes de intervención; para lo comunitario.

Sistema de coordinación desarrollado con servicios de carácter preferente: centros escolares, con el sistema de salud, con promoción/ juventud.

\section{Organización interna}

Tareas de carácter organizativo definidas.

Tiempos y asignación de tareas definidas. Adecuada temporalización de las reuniones.

Coordinación equipo de intervención directa-coordinador (equipo ampliado); equipo de intervención directa- responsable municipal;

entidad- responsable municipal.

Coordinación con representación política.

Reuniones equipo de intervención directa. Reuniones de equipo de equipo ampliado (educadores-coordinador).

Planificación del servicio

Sistema de planificación en base a sus elementos básicos: fundamentación, finalidades, objetivos, programación actuaciones y evaluación. Fundamentada desde el diagnostico de necesidades y el conocimiento exhaustivo de la realidad.

Diseñado según objetivos centrados en las personas.

Programación en coherencia con la planificación general.

Evaluación en proceso en base a fundamentación y objetivos.

Fuente: Elaboración propia. 
del Departamento de Políticas Sociales de Diputación Foral de Gipuzkoa. Este modelo ha servido, igualmente, de referencia para el desarrollo de los procesos de tránsito con algunos municipios de este territorio histórico acompañados por el equipo responsable de dicho programa. El Cuadro 5 recoge el esquema general del modelo.

Como acercamiento a los contenidos del modelo de servicio propuesto, exponemos los elementos que corresponderían a cada bloque de contenido que, desde un punto de vista integral del servicio, se entiende que debe ser en cierto grado definido. Estos elementos desplegados y dotados de contenido conformarían un adecuado desarrollo de cara a la mejora del servicio; en este sentido, sirvan como indicadores y pista que ayuden y orienten a la mejora de la intervención desde los fundamentos que ya se han apuntado a lo largo del artículo:

[...] Me parece necesario desarrollar un modelo orientativo para la implementación del Servicio de Intervención Socioeducativa y Psicosocial (1.3) que defina enfoques, metodologías, etc. y que garantice que el servicio se desarrolla en consonancia con la esencia, los contenidos y los principios de la Ley [...] (Ararteko, 2016: 87).

\section{Algunas recomendaciones para concluir}

Para finalizar esta exposición de aportaciones relacionadas con el encargo que adquieren los servicios sociales municipales en la atención de índole socioeducativa y psicosocial, apuntamos a continuación, a modo de recapitulación, unas recomendaciones de carácter complementario que, igualmente, entendemos que pueden ser útiles para el resto de servicios a implementar o desarrollar.

- Estamos a tiempo de no dar por perdida la oportunidad de revisar nuestros servicios, ver su alcance y reorientarlos hacia una mayor calidad en ellos.

- Del mismo modo, es el momento de asegurar la ubicación funcional de cada uno de los servicios a desarrollar dentro de un único sistema de servicios sociales, ya sea de competencia municipal o foral, y en el marco de fundamentación y coherencia que nos ofrece la actual legislación.
- El papel de la intervención socioeducativa viene definitivamente reforzado e incorporado en el desarrollo de gran parte de los servicios y prestaciones, junto a los y las trabajadoras sociales y el de otras personas profesionales; esto hace preciso delimitar su función específica y su lugar en la tarea compartida.

- La nueva definición de los servicios pasa por el riguroso ajuste de sus propuestas y actuaciones a las necesidades de las personas que es requisito conocer y analizar.

- Parece conveniente establecer un modelo de servicio (1.3) (y modelos) que tenga un carácter unificador y, adaptado a cada realidad municipal, sirva de referencia para su definición.

- El modelo debe garantizar el enfoque centrado en la persona y sus necesidades a partir de un procedimiento de intervención en fases que, con un plan individualizado, permita la atención mediante modalidades suficientes y adecuadas de respuesta; asimismo, fomentar la participación de la persona en sus propios procesos.

- Las dificultades de inclusión en personas adultas son también susceptibles de la respuesta por parte del servicio (1.3), para lo cual, una vez identificadas las personas destinatarias, se deben establecer marcos y modalidades de carácter básico para su atención.

- La intervención como aval de cambio y de transformación debe enfocarse desde la perspectiva comunitaria y a un enfoque que sitúa los servicios sociales como universales y próximos, a partir de las relaciones personalesprofesionales y la contribución al desarrollo comunitario.

- En la medida de lo posible, debe contemplarse la respuesta a las necesidades de las personas desde la corresponsabilidad, para lo cual es necesario establecer sistemas de coordinación que favorezcan la atención compartida.

- El tránsito de las actuaciones, programas o servicios en vigor, puede ser acompañado por procesos de acompañamiento técnico que, desde la aplicación de instrumentos de asesoramiento profesional, herramientas de apoyo y un modelo de referencia de servicio, facilitan la definición de los servicios y el ajuste a las necesidades de las personas. 
ARARTEKO (2016): La situación de los servicios sociales municipales en la Comunidad Autónoma de Euskadi. Situación actual y propuestas de mejora, Serie Informes Extraordinarios, VitoriaGasteiz, Ararteko, «http://www.ararteko.net/ RecursosWeb/DOCUMENTOS/1/o_4074_3.pdf〉.

ÁVILA, A.; HUEGUN, A.; MARTÍN ÁLVAREZ F.; MARTÍN BLÁZQUEZ A. y OTAÑO J. (2017): La intervención socioeducativa en el decreto de cartera del Sistema Vasco de Servicios Sociales. Los Educadores y las Educadoras Sociales en el desarrollo de los Servicios, Comisión para el Desarrollo del Decreto de Cartera de Servicios Sociales de la CAPV, 〈https://www.ceespv.org/ www/attachments/article/924/La intervencion socioeducativa en el decreto de cartera CEESPV-HEFA EHU.pdf〉.

GOBIERNO VASCO (2008): Ley 12/2008, de 5 de diciembre, de Servicios Sociales, Boletín Oficial del País Vasco, 246, 24-12-08, 31.840-31.924, <https:// www.euskadi.eus/r47-bopvapps/es/bopv2/ datos/2008/12/0807143a.shtml.

- (2011): Decreto 230/2011, de 8 de noviembre, por el que se aprueba el instrumento para la valoración de la gravedad de las situaciones de riesgo en los servicios sociales municipales y territoriales de atención y protección a la infancia y adolescencia en la Comunidad Autónoma del País Vasco (BALORA), Boletín Oficial del País Vasco, 223, 12-12-11, <https:// www.euskadi.eus/y22-bopv/es/bopv2/ datos/2011/12/1105937a.shtml.>.

- (2013): Decreto 385/2013, de 16 de julio, por el que se aprueba el Instrumento de Valoración de la Exclusión Social, Boletín Oficial del País Vasco, 149, 7-8-13, 〈https://www.euskadi.eus/y22-bopv/ es/bopv2/datos/2013/08/1303530a.shtmls.
- (2015): Decreto 185/2015, de 6 de octubre, de Cartera de Prestaciones y Servicios del Sistema Vasco de Servicios Sociales", Boletín Oficial del País Vasco, 206, 29-10-15, 1-87, <https:// www.euskadi.eus/y22-bopv/es/bopv2/ datos/2015/10/1504561a.shtml.

- (2017): Decreto 152/2017, de 9 de mayo, por el que se aprueba la actualización del Instrumento para la Valoración de la Gravedad de las Situaciones de Riesgo y Desamparo en los Servicios Sociales Municipales y Territoriales de Atención y Protección a la Infancia y Adolescencia en la Comunidad Autónoma del País Vasco (BALORA), Boletín Oficial del País Vasco, 125, 3-7-17, <https://www.euskadi.eus/y22-bopv/es/ bopv2/datos/2017/07/1703354a.shtml.

LARRIÓN, B.; LETURIA, F.J.; ZALAKAIN, J. y ZABALETA, N. (2018): Elkar-EKIN: (re)construyendo en común la red de servicios sociales para la inclusión en Gipuzkoa, Zerbitzuan, 67, 51-65, <https://doi. org/10.5569/1134-7147.67.04>.

LÓPEZ-AROSTEGI, R. (2016): “Ley de Servicios Sociales y Decreto de Cartera. Aproximación a la intervención socioeducativa en el nuevo escenario de Servicios Sociales" (documentación entregada en la Jornada “La intervención socioeducativa y Decreto de Cartera de Prestaciones y Servicios", Bilbao, Colegio de Educadoras y Educadores Sociales del País Vasco), 〈https://www.ceespv.org/ www/attachments/article/723/Jornada_CES_ Rafa_Lopez_Arostegi.pdf $\rangle$.

MARTÍN ÁLVAREZ, F.; MARTÍN BLÁZQUEZ, A. y OTAÑO, J. (2018): "El proceso colaborativo entre profesionales de la educación y trabajo social y su acompañamiento técnico para la definición de un servicio de intervención socioeducativa 
en el ámbito local", Revista Educación Social, 26, 112-130, <http://www.eduso.net/res/ winarcdoc.php?id=1103>.

OTAÑO, J. (2012): “Nuevas formas de exclusión como consecuencia del fuerte incremento del desempleo. Comparecencia en el Senado ante la Comisión Especial sobre nuevas formas de exclusión social consecuencia del fuerte incremento del desempleo", Revista de Educación Social, 14, 〈http://www.eduso.net/ res/pdf/14/nuefor_res_14.pdf〉.
- (2016): “Intervención socioeducativa desde los servicios sociales de atención primaria. Cómo, con quién y para qué", en VV.AA., $V$ Informe sobre la situación de los Servicios Sociales en Euskadi, Consejo Vasco de Servicios Sociales, 42- 58, 〈https://www.irekia.euskadi.eus/ uploads/attachments/8350/V_Informe_CVSS. pdf?1467805283'. 